Rivista di matematica per le scienze economiche e sociali - Anno $17^{\circ}$, Fascicolo $2^{\circ}$

\title{
SULLA VITALITÀ DI UN MERCATO FINANZIARIO*
}

\author{
GIANCARLO COSTA
}

Università "L. Bocconi", Milano

MARCO LI CALZI

Università “Ca' Foscari”, Venezia

Versione definitiva pervenuta il $28 / 03 / 95$

Questo lavoro discute un modello di mercato finanziario basato sulla teoria della vitalità associata allo studio delle inclusioni differenziali. Il modello permette di esplicitare l'esistenza di una relazione di dipendenza tra le quantità di un titolo detenute dagli agenti ed il suo prezzo di mercato. Basandosi su questa, è possibile ottenere rigorosamente l'esistenza di un sentiero temporale per il prezzo senza bisogno di introdurre ipotesi ad hoc.

\section{Introduzione}

Larga parte della moderna teoria della finanza si basa sull'ipotesi di mercati perfetti ed efficienti. Una conseguenza di ciò è che lo studio dei prezzi dei titoli in un mercato finanziario tende a privilegiare l'importanza delle cosiddette relazioni "fondamentali", come il flusso atteso di cassa e le preferenze degli investitori.

La ricerca più recente sulla microstruttura dei mercati finanziari, tuttavia, ha messo in luce altri fattori che concorrono a determinare l'evoluzione dei prezzi. Tra questi ricordiamo i vincoli istituzionali, le asimmetrie informative ed il comportamento degli investitori. Questo lavoro si concentra proprio su quest'ultimo elemento.

Come è discusso lucidamente in Beja e Goldman (1980), l'importanza del comportamento degli investitori discende da un'osservazione semplice ma cruciale sulla differenza tra il reale funzionamento di un mercato ed il modello walrasiano. Secondo questo, il processo di tatonnement necessario per portare un mercato in equilibrio ha luogo in un tempo virtuale in cui non c'è scambio. Le

* Ringraziamo E. Castagnoli per alcuni utili commenti. Le conversazioni con J.-P. Aubin, F. Moriconi e F. Ortu sull'etimologia e l'interpretazione dei concetti di vitalità ci sono state di grande beneficio. 
transazioni hanno luogo soltanto dopo che il banditore ha "trovato" un prezzo che eguaglia la domanda e l'offerta.

Nella realtà, al contrario, gli investitori sono liberi di comprare e vendere anche durante gli stati di disequilibrio in cui il prezzo è troppo alto o troppo basso. Gli agenti possono quindi cercare di approfittare delle opportunità di guadagno che emergono fuori dall'equilibrio. Cosi facendo, le loro azioni possono far permanere o persino aggravare lo stato di disequilibrio, generando un andamento del prezzo (almeno temporaneamente) slegato dalle relazioni fondamentali.

La letteratura ha elaborato numerosi modelli di questa situazione, che per semplicità suddividiamo in due classi. Nella prima possiamo collocare i modelli che ottengono esplicitamente il processo di evoluzione del prezzo dal comportamento massimizzante dei singoli agenti. Al costo di una formalizzazione complessa e di ipotesi molto forti, questi modelli cercano di offrire un fondamento microeconomico rigoroso al concetto mesoeconomico di andamento del mercato. Vedi tra gli altri Benabou e Laroque (1992), De Long et alii (1990, 1991) e Welch (1992).

Nella seconda classe possiamo raccogliere i modelli che preferiscono descrivere direttamente il processo di evoluzione del prezzo giustificando soltanto per via intuitiva le equazioni utilizzate a tale scopo. Nonostante (o forse in virtù di) questa semplicità, ci sembra che tali modelli riescano a catturare più da vicino i punti cruciali del problema. Vedi tra gli altri Antoci e Barucci (1993), Beja e Goldman (1980), Bulow e Klemperer (1994), Caplin e Lehay (1992), Cenci e Cerquetti (1991), Day e Huang (1990), De Long et alii (1990a), Ghezzi (1992) ed i lavori del gruppo di Torino raccolti in Cornaglia et alii (1991).

Pur se più convincenti, i modelli della seconda classe non sono esenti da critiche. Tra queste, due sono di particolare interesse ai fini di questo lavoro. Entrambe si possono ricondurre all'enfasi che i modelli di questa classe pongono sulla costruzione di un sistema dinamico in cui la variabile di stato è il prezzo di mercato, anziché le quantità del titolo possedute da ciascun agente. L'equilibrio di un mercato si fonda sull'eguaglianza delle quantità offerte e domandate. Esse dovrebbero determinare l'evoluzione del prezzo, e non viceversa.

La prima critica, di natura teorica, pone il problema di giustificare la semplificazione inerente all'uso del solo prezzo anziché delle quantità possedute da tutti gli agenti come variabile di stato. Più precisamente, ci si chiede se esistano condizioni sufficienti sulle quantità domandate o offerte che garantiscono l'esistenza di un sentiero temporale del prezzo, eventualmente individuato mediante un sistema dinamico.

La seconda critica, di natura tecnica, pone il problema della robustezza di questi modelli rispetto alle diverse specificazioni della legge che governa l'evoluzione del prezzo. Molti modelli, infatti, ipotizzano una forma funzionale ad hoc per il sistema dinamico del prezzo e basano le loro conclusioni sulle proprietà di questo. La questione è ancora più delicata se, come già osservato, si considera che l'evoluzione del prezzo dovrebbe essere posta in relazione con gli eccessi di domanda.

In questo lavoro presentiamo un approccio che offre una risposta che ci pare adeguata ad entrambe queste critiche. L'approccio si basa sulla teoria delle inclusioni differenziali e fa riferimento in particolare alla teoria della vitalità per i sistemi dinamici, che studia il seguente problema. Data la descrizione 
matematica di un sistema fisico o economico, quali condizioni garantiscono che il modello non ammetta tra le sue soluzioni traiettorie per le quali non è possibile dare un'interpretazione significativa? In altre parole, come possiamo garantire che un modello matematico parli solo di ciò che cerca di descrivere?

La teoria della vitalità su cui si basa questo lavoro è quella sviluppata da Aubin e dai suoi collaboratori, nota in lingua anglosassone come viability. Questo termine, che anglicizza il vocabolo francese viabilité derivato da vie, ha la connotazione di "capacità del sistema di mantenersi vivo". Nel caso dei mercati finanziari, tuttavia, esso si presta alla confusione con un'altra accezione di viability, che grosso modo designa l'ipotesi secondo la quale non possono esistere strategie in grado di arricchire senza limiti un individuo. Si veda ad esempio Harrison e Kreps (1979). Questa seconda denotazione ha origine dal latino via e suggerisce l'idea che il sistema rimanga su un "sentiero percorribile".

Le due accezioni del termine, pur se simili, si riferiscono a contesti modellistici diversi. Ci è parso utile, pertanto, scrivere questo lavoro in lingua italiana per suggerire l'adozione di una terminologia tecnica che le distingua. In particolare, tradurremo viabilité con "vitalità" e viability con "viabilità". 1

Più precisamente, nella sezione 1 discutiamo un modello di mercato basato sulle quantità possedute dagli agenti e mostriamo come garantire l'esistenza di un sentiero temporale per il prezzo basandoci su ipotesi molto generali sulle funzioni di domanda. Nella sezione 2, approfondiamo l'analisi del modello sotto ipotesi più specifiche che consentono di definire una relazione tra prezzo e quantità del titolo possedute dagli agenti. Nella sezione 3, infine, utilizziamo questi risultati per offrire un esempio "robusto" di comportamento adattivo. Chiude il lavoro un'appendice in cui si raccolgono definizioni e risultati mutuati dalla teoria della vitalità.

\section{Esistenza di un prezzo coerente}

Consideriamo un mercato azionario dove si scambia un solo titolo infinitamente divisibile. Il mercato è senza frizioni e gli scambi avvengono in tempo continuo. Supponiamo che sul mercato siano presenti $n$ agenti, ciascuno dei quali detiene una quantità $x_{i} \in[0,1]$ del titolo $(i=1, \ldots, n)$. Ponendo per convenzione che la quantità disponibile del titolo sia 1 , imponiamo che $\sum_{i=1}^{n} x_{i}=1$. Indichiamo con $p$ il prezzo unitario del titolo. Questo prezzo può variare in un intervallo compatto (e non degenere) contenuto in $\mathbf{R}^{+}$, che senza perdita di generalità poniamo uguale a $I=[0,1]$.

Ad ogni istante $t \in[0,+\infty)$, pertanto, la situazione del mercato è descritta da un vettore di quote di investimento $x(t)=\left(x_{1}(t), \ldots, x_{n}(t)\right)$ nel simplesso unitario $S^{n}$ e da un prezzo $p(t)$ in $[0,1]$. Ogni agente può modificare in qualsiasi momento la sua dotazione $x_{i}(t)$ in funzione della situazione del mercato. Un metodo tradizionale per rappresentare questa possibilità consiste nel supporre che in ogni istante $t$ l'evoluzione del mercato sia descritta dal sistema di equazioni differenziali

1 Traduzioni alternative suggerite nelle nostre conversazioni con amici e colleghi sono rispettivamente "vivibilità" e "percorribilità". 


$$
\left\{\begin{array}{l}
x_{i}^{\prime}(t)=d_{i}\left(x_{i}(t), p(t)\right) \\
x(0)=\left(x_{1}(0), \ldots, x_{n}(0)\right) \in S^{n}
\end{array} \quad i=1, \ldots, n\right.
$$

dove $d_{i}:[0,1] \times[0,1] \rightarrow[-1,1]$ indica la funzione di domanda istantanea dell'agente $i$, per ogni $i=1, \ldots, n$. Si noti che, conformemente all'esistenza di vincoli istituzionali sulle possibilità di acquisto e vendita, abbiamo supposto che il codominio delle domande istantanee sia un intervallo compatto: in particolare, ma senza perdita di generalità, l'intervallo $[-1,1]$.

$\grave{E}$ evidente che la (1) presuppone l'esistenza di un sentiero temporale $p(t)$ per il prezzo. La funzione $p(t)$, tuttavia, non può essere scelta liberamente. In ogni istante $t$, infatti, il mercato descritto dalla (1) deve soddisfare una condizione di equilibrio che impone l'eguaglianza tra le quantità scambiate. In termini matematici, per ogni $t \geq 0$ occorre che

$$
\sum_{i=1}^{n} d_{i}\left(x_{i}(t), p(t)\right)=0
$$

Questa condizione di coerenza, insieme alla (1), definisce "implicitamente" la funzione $p(t)$. Come già discusso nell'introduzione, sorge allora il problema di dimostrare l'esistenza (ed in subordine l'unicità) di un sentiero temporale coerente del prezzo.

A tale scopo, è utile riformulare la (1) in modo da non impegnarsi a priori sull'esistenza di $p(t)$. Riscriviamo quindi la (1) come un sistema di inclusioni differenziali

$$
\left\{\begin{array}{l}
x_{i}^{\prime}(t) \in d_{i}\left(x_{i}(t), I\right) \\
x(0)=\left(x_{1}(0), \ldots, x_{n}(0)\right) \in S^{n}
\end{array} \quad i=1, \ldots, n\right.
$$

in cui abbiamo sostituito alla funzione $p(t)$ il suo codominio.

Data una configurazione delle quantità possedute $x \in S^{n}$, indichiamo con

$$
C(x)=\left\{p \in[0,1] \mid \sum_{i=1}^{n} d_{i}(x, p)=0\right\}
$$

l'insieme dei prezzi che consentono alla (2) di soddisfare la condizione di coerenza e chiamiamo la corrispondenza $C: S^{n} \rightarrow[0,1]$ cosi definita mappa di coerenza del prezzo.

L'esistenza di un sentiero temporale coerente per il prezzo equivale a richiedere che per ogni istante $t \geq 0$ esista una selezione $p(t) \in C(x(t))$. Poiché possiamo scegliere arbitrariamente la condizione iniziale in $S^{n}$, condizione necessaria perché ciò avvenga è che $C(x) \neq \emptyset$ per ogni $x \in S^{n}$. Inoltre, poiché il valore di $p(t)$ nell'istante $t$ influenza la domanda $x_{i}^{\prime}(t)$ e quindi la quantità posseduta da ciascun agente $i$, occorre anche garantire che il prezzo $p(t)$ non sospinga $x(t)$ fuori dal simplesso $S^{n}$, rendendo priva di significato la mappa di coerenza.

In termini matematici, possiamo formulare il problema dell'esistenza di un sentiero temporale coerente del prezzo come segue. Diciamo che una soluzione 
$x$ della (2) è vitale in $S^{n}$ se $x(t) \in S^{n}$ per ogni $t \geq 0$. Osservando che nella (1) l'evoluzione di $p$ "controlla" l'evoluzione delle variabili di stato sintetizzate nel vettore $x$, l'esistenza nella (2) di un sentiero $p(t)$ coerente equivale all'esistenza di un controllo $p$ che genera una soluzione $x(t)$ vitale in $S^{n}$ tale che $p(t) \epsilon$ $C(x(t))$ per ogni $t \geq 0$. Il teorema seguente mostra che tale problema ammette soluzione sotto ipotesi di regolarità molto naturali. In particolare, si noti che le ipotesi sulle funzioni di domanda degli agenti sono estremamente generali. Per la dimostrazione, si veda anche Teorema 5.4.1 in Aubin e Cellina (1984), p. 239.

TEOREMA 1. Supponiamo che per ogni $i=1, \ldots, n$ le funzioni di domanda $d_{i}\left(x_{i}, p\right)$ nella (2) siano continue in entrambi gli argomenti e tali che

$$
d_{i}(0, p) \geq 0 \quad \text { e } \quad d_{i}(1, p) \leq 0
$$

per ogni $p$ in $[0,1]$ ed ogni $i=1, \ldots, n$. Inoltre, supponiamo che la mappa di coerenza del prezzo $C$ definita dalla (3) sia tale che

$$
C(x) \neq \emptyset
$$

per ogni $x$ in $S^{n}$. Allora, per ogni condizione iniziale $x(0)=x_{0} \in S^{n}$, l'inclusione differenziale (2) ammette una soluzione $x$ vitale in $S^{n}$ alla quale è associata una funzione di controllo misurabile $p^{*}(t) \in C(x(t))$ tale che $x$ e $p^{*}$ soddisfano il sistema di equazioni differenziali (1).

Dimostrazione. Ponendo $F(x)=\prod_{i=1}^{n} d_{i}\left(x_{i}(t),[0,1]\right)$ e $K=S^{n}$, riscriviamo la (2) in forma più compatta come

$$
\left\{\begin{array}{l}
x^{\prime}(t) \in F(x(t)) \\
x(0)=x_{0} \in K
\end{array}\right.
$$

ed applichiamo il teorema di vitalità enunciato in appendice. Poiché le ipotesi (4) e (5) implicano la (14), questo teorema garantisce l'esistenza di una soluzione vitale $x$ in $S^{n}$ per ogni $t \in[0,+\infty)$; ovvero, tale che per quasi ${ }^{2}$ ogni $t \geq 0$

$$
x^{\prime}(t) \in \prod_{i=1}^{n} d_{i}\left(x_{i}(t),[0,1]\right)
$$

$\mathrm{e}$

$$
\sum_{i=1}^{n} x_{i}^{\prime}(t)=0
$$

Ci resta da dimostrare che esiste un controllo coerente $p(t)$. In virtù del Corollario 1.14.1 in Aubin e Cellina (1984), p. 90, la (6) implica l'esistenza di una selezione misurabile $p^{*}:[0,+\infty) \rightarrow[0,1]$ tale che

$$
x^{\prime}(t)=\prod_{i=1}^{n} d_{i}\left(x_{i}(t), p^{*}(t)\right)
$$

2 Ricordiamo che la soluzione di un'inclusione differenziale è definita a meno di un insieme di misura nulla. 
per quasi ogni $t \geq 0$. Congiuntamente alla (7), questo implica

$$
p^{*}(t) \in C(x(t))
$$

per quasi ogni $t \geq 0$ e dimostra l'esistenza quasi ovunque di un sentiero del prezzo coerente.

Per estendere l'esistenza ad ogni $t \geq 0$, è sufficiente ridefinire $p^{*}(t)$ sull'insieme di misura nulla in cui eventualmente non valga la (9) osservando che questo non altera la (8) e quindi non modifica la soluzione vitale già trovata.

Osserviamo che, in base al teorema, la dipendenza della funzione di controllo $p^{*}$ dal tempo è mediata dalla sua dipendenza dalle quantità possedute dagli agenti in ogni istante e quindi che la sua evoluzione è direttamente influenzata dallo stato del sistema.

\section{Continuità e unicità del prezzo}

In questa sezione arricchiamo il modello introdotto nella sezione precedente, specificando alcune plausibili caratteristiche qualitative per la forma delle funzioni di domanda degli agenti. In particolare, introduciamo l'ipotesi consueta in letteratura che l'eccesso di domanda di un agente dipenda anche dal valore "fondamentale", ovvero di lungo periodo, che questi attribuisce al titolo. In proposito, si veda ad esempio Day e Huang (1990).

Indichiamo pertanto con $u_{i} \in[0,1]$ la stima del valore fondamentale del titolo fatta da ogni agente $i=1, \ldots, n$ sulla base della sua informazione. Poiché agenti diversi hanno in generale stime diverse, non richiediamo che $u_{i}=u_{j}$ per $i \neq j$. Dato un prezzo corrente $p$, supponiamo che la domanda istantanea dell'agente $i$ abbia lo stesso segno della differenza $u-p$. Questa ipotesi molto naturale discende dall'osservazione che un agente trova vantaggioso vendere o comprare a seconda che rispettivamente ritenga il prezzo del titolo sovra o sottostimato.

Nel nostro modello, dove la domanda dipende sia dal prezzo sia dalla quantità posseduta del titolo, occorre specificare anche in che modo queste due variabili interagiscano. A questo proposito, ci limitiamo alle seguenti ipotesi molto generali, che supponiamo valide per ogni agente $i=1, \ldots, n$ :

1. La domanda istantanea $d_{i}\left(x_{i}, p\right)$ è funzione strettamente decrescente della quantità posseduta per ogni $p \in[0,1]$.

2. La valutazione del valore fondamentale del titolo non dipende dalla quantità posseduta; ovvero, esiste $u_{i} \in[0,1]$ tale che $d_{i}\left(x_{i}, u_{i}\right)=0$ per ogni $x_{i} \in[0,1]$.

3. Supponi $x_{i}=0$. Se $p<u_{i}$, la domanda istantanea $d_{i}(0, p)>0$ è strettamente decrescente nel prezzo; se $p \geq u_{i}, d_{i}(0, p)=0$.

4. Supponi $x_{i}=1$. Se $p>u_{i}, \overline{d_{i}}(1, p)<0$ è strettamente decrescente nel prezzo; se $p \leq u_{i}, d_{i}(1, p)=0$.

5. Infine, se $x_{i} \in(0,1), d_{i}\left(x_{i}, p\right)<0$ è strettamente decrescente nel prezzo con $d_{i}\left(x_{i}, 0\right)>0$ e $d_{i}\left(x_{i}, 1\right)<0$.

6. Le funzioni di domanda istantanee sono continue in entrambi gli argomenti.

Per facilitare la lettura di queste ipotesi, abbiamo rappresentato in Figura 1 il grafico tipico di una funzione di domanda istantanea rispetto al prezzo nei tre casi $x_{i}=0, x_{i} \in(0,1)$ e $x_{i}=1$. 


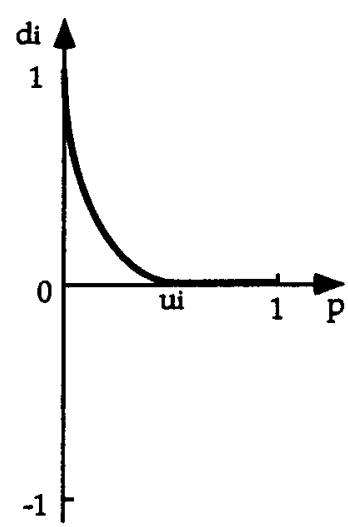

$\mathrm{xi}=0$

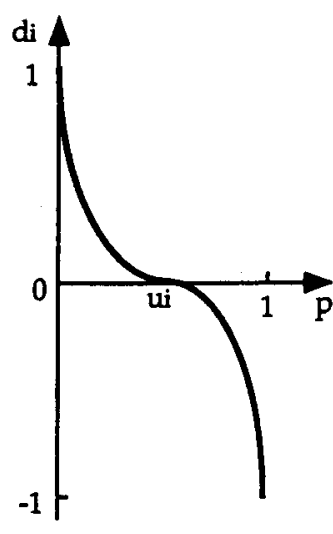

$0<x i<1$

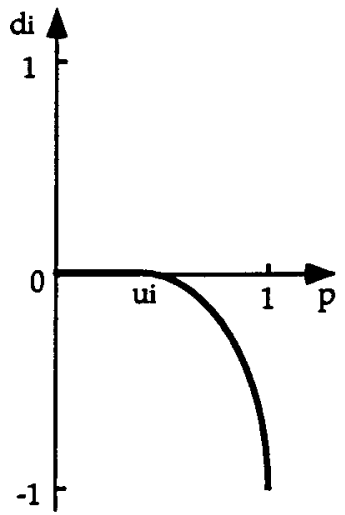

$\mathrm{xi}=1$

Fig. 1 Funzioni di domanda istantanee.

Si noti che la domanda $d_{i}\left(x_{i}, p\right)$ dell'agente $i$ è sempre una funzione strettamente decrescente del prezzo, tranne due casi in cui è soltanto (debolmente) decrescente: a) $x_{i}=0$ e $p \geq u_{i}$; b) $x_{i}=1$ e $p \leq u_{i}$. Queste due eccezioni sono necessarie per non imporre che un agente si impegni in uno scambio che peggiora la sua situazione. Infatti, nel primo caso il prezzo corrente $p$ è superiore alla valutazione del valore fondamentale $u_{i}$ e pertanto l'agente preferisce mantenere una dotazione nulla del titolo; nel secondo caso, viceversa, $p$ è inferiore a $u_{i}$ e l'agente preferisce mantenere la proprietà di tutto il titolo.

Un'importante conseguenza di ciò è la seguente. Senza perdita di generalità, ribattezziamo gli agenti in modo che le loro valutazioni fondamentali siano ordinate come $u_{1} \geq u_{2} \geq \ldots \geq u_{n}$. Allora la funzione di domanda aggregata istantanea $D(\bar{x}, p)=\sum_{i=1}^{n} \bar{d}_{i}\left(x_{i}, p\right)$ è sempre strettamente decrescente nel prezzo, tranne un caso in cui è soltanto (debolmente) decrescente: $u_{1}>u_{2}$ e $x_{1}=1$. Si noti che se $u_{1}=u_{2}$, la decrescenza stretta nel prezzo vale comunque. Per unificare la notazione, denotiamo con $e_{1}=(1,0, \ldots, 0)$ il vertice del simplesso con la prima coordinata uguale a 1 e poniamo $\tilde{S}^{n}=S^{n} \backslash\left\{e_{1}\right\}$ se $u_{1}>u_{2}$ e $\tilde{S}^{n}=S^{n}$ se $u_{1}=u_{2}$. Allora $D(x, p)$ è funzione strettamente decrescente del prezzo per ogni $x \in \tilde{S}^{n}$. Inoltre, se $x=e_{1}$ e $u_{1}>u_{2}$, la condizione di coerenza del prezzo impone che $p \in\left[u_{2}, u_{1}\right]$ e in questo caso non c'è scambio.

Il seguente teorema mostra che le nostre ipotesi sulle funzioni di domanda degli agenti sono sufficienti per affermare che su $\tilde{S}^{n}$ la mappa di coerenza del prezzo si riduce ad una funzione, per di più continua.

TEOREMA 2. Sotto le ipotesi 1-6, la restrizione a $\tilde{S}^{n}$ della mappa di coerenza del prezzo definita dalla (3) per l'inclusione differenziale (2) ̀̀ una funzione continua. 
Dimostrazione. Per ogni $x \in \tilde{S}^{n}$, la domanda aggregata istantanea $D(x, p)$ è una funzione continua e strettamente decrescente in $p$ con $D(x, 0) \geq 0$ e $D(x, 1) \leq 1$. Ne segue che, per ogni $x \in \tilde{S}^{n}$, esiste un unico valore $\hat{p}=$ $\hat{p}\left(x_{1}, \ldots, x_{n}\right)$ tale che $D(x, \hat{p})=0$. La mappa di coerenza del prezzo coincide con la funzione $\hat{p}: \tilde{S}^{n} \rightarrow[0,1]$ cosi definita.

Ci resta da dimostrare che $\hat{p}$ è anche continua. Per farlo, si osservi che la mappa di coerenza $C(x)$ è una corrispondenza superiormente semicontinua, perché ha grafico chiuso e codominio compatto. Poiché la funzione $\hat{p}(x)$ non $\dot{\mathrm{e}}$ altro che la restrizione di $C(x)$ a $\tilde{S}_{n}$, la superiore semicontinuità della mappa implica la continuità della funzione.

Secondo questo teorema, finché $x \in \tilde{S}^{n}$, l'unica selezione della mappa $C(x)$ è la funzione continua $\hat{p}(x)$. Questo suggerisce di rileggere il controllo coerente (e in anello aperto) $p(t)$ associato ad una particolare soluzione vitale $x^{*} \mathrm{di}$

$$
\left\{\begin{array}{l}
x_{i}^{\prime}(t) \in d_{i}\left(x_{i}(t),[0,1]\right) \\
x(0)=\left(x_{1}(0), \ldots, x_{n}(0)\right) \in S^{n}
\end{array} \quad i=1, \ldots, n\right.
$$

come l'unico controllo in anello chiuso $\hat{p}(x(t))$ tale che

$$
\left\{\begin{array}{l}
x_{i}^{\prime}(t)=d_{i}\left(x_{i}(t), \hat{p}\left(x_{1}(t), \ldots, x_{n}(t)\right)\right) \quad i=1, \ldots, n \\
x(0)=\left(x_{1}(0), \ldots, x_{n}(0)\right) \in S^{n}
\end{array}\right.
$$

ammetta la soluzione vitale $x^{*}$ in $S^{n}$. In altre parole, si tratta di mostrare che per ogni soluzione vitale l'evoluzione del prezzo nel tempo è univocamente influenzata dallo stato del sistema.

Se $\hat{p}$ fosse definita su $S^{n}$, questa rilettura sarebbe immediata. Tuttavia, poiché il suo dominio è più piccolo, dobbiamo accontentarci di un risultato (solo apparentemente) più debole. Poiché il caso $u_{1}=u_{2}$ non pone problemi, supponiamo $u_{1}>u_{2}$ e definiamo per ogni soluzione $x$ della (14) l'istante

$$
T_{x}=\inf \left\{t \geq 0 \mid x(t)=e_{1}\right\}
$$

Per $t<T_{x}$, sia $x(t)$ sia $\hat{p}(x(t))$ sono ben definiti.

In termini matematici, le difficoltà sorgono quando $T_{x}<+\infty$, perché il controllo $\hat{p}(x(t))$ non è più una funzione per $t \geq T_{x}$. Tuttavia, in termini economici, questo ha una facile spiegazione che consente di risolvere la difficoltà. In $t=T_{x}$, $\boldsymbol{x}(t)=e_{1}$ : non c'è più scambio ed il prezzo può variare nell'intervallo $\left[\boldsymbol{u}_{2}, u_{1}\right]$. Inoltre, questa situazione permane anche per $t>T_{x}$. Nessuno degli agenti domanda o offre alcunché e quindi il prezzo risulta indeterminato (ma anche economicamente irrilevante) in $\left[u_{2}, u_{1}\right]$.

Se il controllo $p(t) \in\left[u_{2}, u_{1}\right]$ per ogni $t \geq T_{x}$, il percorso della soluzione resta $x(t)=e_{1}$ da $T_{x}$ in avanti. Quindi, per recuperare l'unicità del controllo, è sufficiente prolungarlo per continuità imponendo arbitrariamente

$$
\hat{p}(x(t))=\lim _{t \rightarrow T_{x}} \hat{p}(x(t)) \quad \text { per } t \geq T_{x}
$$


ovvero, qualora il limite non esista, fissando a piacere per $\hat{p}(x(t))$ un valore (ad esempio, $u_{1}$ ) nell'intervallo $\left[u_{1}, u_{2}\right]$ per ogni $t \geq T_{x}$. Si noti che la definizione di questa funzione dipende da $T_{x}$ e quindi dalla soluzione $x$ a cui si applica il controllo. In particolare, questa definizione non risolve il caso in cui la condizione iniziale sia $x_{0}=e_{1}$, corrispondente ad un mercato virtualmente inesistente.

Con la qualificazione $x_{0} \in \tilde{S}^{n}$, quindi, possiamo concludere che ogni soluzione vitale della (10) corrisponde ad una soluzione della (11) regolata dalla funzione di controllo $\hat{p}$ continua ed in anello chiuso.

\section{Un esempio}

Nella sezione precedente abbiamo supposto che le valutazioni dei valori fondamentali $u_{1}, \ldots, u_{n}$ restassero costanti nel tempo. '̀ verosimile, tuttavia, che nel tempo queste siano influenzate dall'arrivo di nuova informazione o dall'osservazione dei comportamenti altrui. In questa sezione presentiamo un modello semplice ma "robusto" di aggiustamento adattivo per le valutazioni dei valori fondamentali. Per comodità, continuiamo a supporre valide le ipotesi 1-6 della sezione precedente.

Ipotizziamo che ogni agente $i$ riveda la sua valutazione $u_{i}$ del valore fondamentale del titolo negli istanti $\tau_{1}^{i}, \ldots, \tau_{k}^{i}, \ldots$ Per semplicità, ma senza rilevante perdita di generalità, supponiamo anche che $\tau_{k+1}^{i}-\tau_{k}^{i}=\tau$ per ogni agente $i$ ed ogni $k=1,2, \ldots$, di modo che tutti gli agenti aggiornino la loro valutazione contemporaneamente negli istanti $\tau, 2 \tau, \ldots$.

L'ipotesi che in un modello in tempo continuo come il nostro gli agenti rivedano le loro valutazioni o più in generale il loro comportamento in tempo discreto non è nuova. Vedi Cenci e Cerquetti (1991) o i lavori in Cornaglia et alii (1991). La sua giustificazione tradizionale è che il processo di raccolta ed elaborazione dell'informazione richiede tempo ed è costoso.

Ci saranno utili alcune osservazioni. L’ipotesi che gli agenti rivedano le loro valutazioni implica che la dinamica del sistema si aggiusti istantaneamente ogni $\tau$ istanti e "riparta". I teoremi sulla vitalità del sistema, quindi, vanno riletti rispetto all'orizzonte temporale $[k \tau,(k+1) \tau)$ invece che rispetto a $[0,+\infty)$.

Inoltre, come è ovvio, non ha più senso supporre $u_{1} \geq u_{2} \geq \ldots \geq u_{n}$ perché l'ordinamento delle valutazioni degli agenti può cambiare. Indichiamo quindi con $u^{-}(t)=\min _{i} u_{i}(t)$ e $u^{+}(t)=\max _{i} u_{i}(t)$. Le ipotesi $1-6$ implicano che la funzione di controllo $\hat{p}(x)$ del Teorema 2 nel periodo $[k \tau,(k+1) \tau)$ appartiene all'intervallo $\left[u^{-}(k \tau), u^{+}(k \tau)\right]$, purché la condizione iniziale del periodo non sia $x(\tau)=e_{k}$ dove $k$ è l'(unico) agente per cui $u_{k}(\tau)=u^{+}(\tau)$. Escludiamo per ipotesi questo caso economicamente irrilevante.

Per modellare l'ipotesi che gli agenti aggiustino le loro valutazioni dei valori fondamentali in modo adattivo, supponiamo che per ogni agente $i$ ed ogni $k=$ $1,2, \ldots$ sia

$$
u_{(k+1) \tau}^{i}=u_{k \tau}^{i}-\alpha_{k}^{i}\left[u_{k \tau}^{i}-\hat{p}((k+1) \tau)\right]
$$

dove $\alpha_{k}^{i} \in(0,1]$ rappresenta la velocità di aggiustamento $\mathrm{e}$

$$
\hat{p}((k+1) \tau)=\lim _{t \uparrow(k+1) \tau} \hat{p}(x(t))
$$


Si noti che ogni agente utilizza "l'ultimo" prezzo del periodo precedente per generare la valutazione del periodo successivo.

Sotto l'ipotesi addizionale che gli agenti non ricevano informazioni esogene, la (12) equivale a supporre che gli agenti si comportino come se il prezzo corrente riassumesse in modo efficiente l'informazione espressa dal mercato. Si noti che la naturalezza di questa interpretazione richiede che il prezzo dipenda effettivamente dallo stato $x(t)$ del sistema, ovvero che possa essere espresso come un controllo in anello chiuso. Per questo motivo, nella (12) consideriamo $\hat{p}$ anziché $p$.

Se tutti gli agenti aggiornano le loro valutazioni fondamentali secondo la (12) la differenza $u^{+}(t)-u^{-}(t) \rightarrow 0$ per $t \rightarrow+\infty$. Ne segue che il sentiero temporale $p(t)$ del prezzo può essere descritto come un controllo in anello chiuso $\hat{p}(x(t))$ che: a) varia con continuità sull'intervallo $[k \tau,(k+1) \tau)$ per ogni $k=1,2, \ldots$; b) può presentare discontinuità nei punti $t=k \tau$, per $k=1,2, \ldots ;$ c) converge ad un valore

$$
\bar{p}=\lim _{t \rightarrow+\infty} u^{+}(t)=\lim _{t \rightarrow+\infty} u^{-}(t)
$$

per $t \rightarrow+\infty$.

La figura 2 riporta una possibile traiettoria del prezzo che illustra queste caratteristiche. Il mercato subisce shock periodici di impatto decrescente in cui gli agenti riallineano le loro opinioni. Nel tempo, le valutazioni del valore fondamentale convergono a $\bar{p}$, che emerge asintoticamente come il valore fondamentale di mercato.

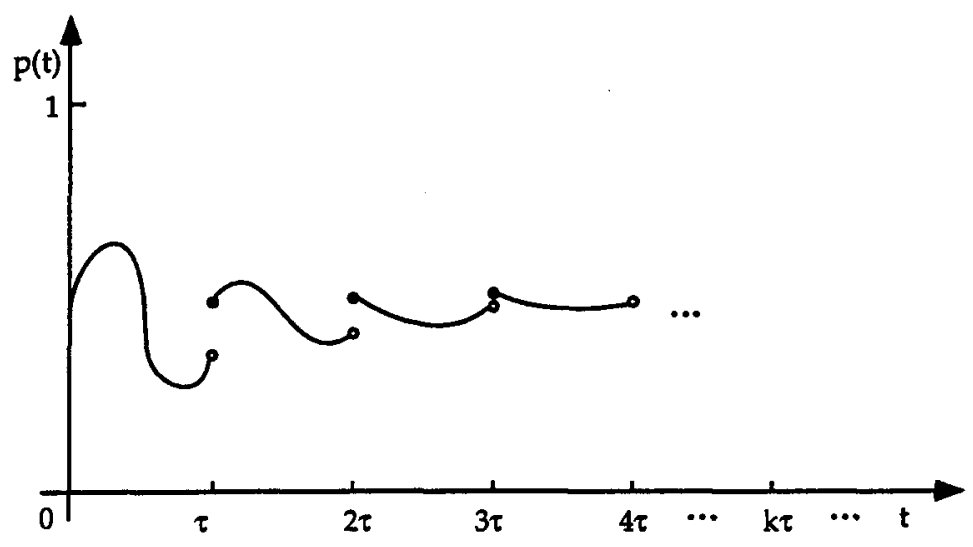

Fig. 2 Una possibile traiettoria del prezzo.

\section{Appendice}

Data una corrispondenza $F: \mathbf{R}^{n} \rightarrow \mathbf{R}^{n}$, consideriamo l'inclusione differenziale

$$
\left\{\begin{array}{l}
x^{\prime}(t) \in F(x(t)) \\
x(0)=x_{0}
\end{array}\right.
$$


Dato un intervallo $J \subset \mathbf{R}$ ed un sottoinsieme chiuso $K \subset \mathbf{R}^{n}$, diremo che una traiettoria $x: J \rightarrow \mathbf{R}^{n}$ per l'inclusione differenziale (13) è vitale in $K$ su $J$ se $x(t) \in K$ per ogni $t \in J$. La condizione di vitalità di una traiettoria, dunque, esprime la proprietà che questa non abbandoni la regione $K$.

Un risultato importante nella teoria delle inclusioni differenziali fornisce un insieme di condizioni sufficienti per l'esistenza di traiettorie vitali. Prima di enunciarlo, richiamiamo alcune definizioni.

Data una corrispondenza $F: \mathbf{R}^{n} \rightarrow \mathbf{R}^{n}$, diciamo che $F$ è superiormente semicontinua in un punto $x_{0}$ del suo dominio se per ogni intorno $N$ di $F\left(x_{0}\right)$ esiste un intorno $M$ di $x_{0}$ tale che $F\left(M\left(x_{0}\right)\right) \subset N$. Diciamo inoltre che $F \dot{\mathrm{e}}$ superiormente semicontinua se è superiormente semicontinua in ogni punto del suo dominio.

Dato un sottoinsieme chiuso $K \subset \mathbf{R}^{n}$, chiamiamo cono contingente di Bouligand in $x$ per $K$ l'insieme

$$
V_{K}(x)=\left\{v \in \mathbf{R}^{n} \mid \liminf _{h \rightarrow 0^{+}} \frac{d_{K}(x+h v)}{h}=0\right\}
$$

dove $d_{K}(x)=\inf _{y \in K} d(x, y)$ denota la distanza del punto $x$ dall'insieme $K$.

Siamo adesso pronti per enunciare il teorema di vitalità. Per la sua dimostrazione (in un caso più generale del nostro), si veda Teorema 4.2.1 in Aubin e Cellina (1984), p. 180.

TEOREMA 3. [Teorema di vitalità] Siano $F: \mathbf{R}^{n} \rightarrow \mathbf{R}^{n}$ una corrispondenza superiormente semicontinua con immagini compatte e convesse $e K$ un sottoinsieme chiuso del suo dominio. Se

$$
F(x) \cap V_{K}(x) \neq \emptyset \quad \text { per ogni } x \in K
$$

allora per ogni condizione iniziale $x_{0} \in K$ esiste un $T>0$ tale che (13) possiede almeno una soluzione vitale in $K$ su $[0, T)$. Inoltre, se $K$ è compatto oppure $F(K)$ è limitato, possiamo prendere $T=+\infty$.

Si noti che $V_{K}(x)=\mathbf{R}^{n}$ per ogni punto $x$ interno a $K$. La (14), quindi, coinvolge in realtà soltanto i punti appartenenti alla frontiera di $K$ ed esprime intuitivamente la proprietà che per ciascuno di tali punti esista almeno una velocità che sospinge il sistema verso "l'interno" di $K$.

\section{BIBLIOGRAFIA}

[1] ANTOCI A., BARUCCI E., A dynamic stock market model with heterogeneous traders and learning, XIV Meeting of the Euro Working Group on Financial Modelling, Mantova, novembre 1993.

[2] AUBIN J.-P., Evolution of viable allocations, J. of Econ. Behav. and Org., 16, $1991,183-215$.

[3] AUBIN J.-P., Viability Theory, Birkhäuser, Boston, 1991. 
[4] Aubin J.-P., Cellina A., Differential Inclusions, Springer-Verlag, Berlin, 1984.

[5] BeJA A., Goldman M. B., On the dynamic behavior of prices in disequilibrium, J. of Fin., 35, 1980, 235-248.

[6] BENABOU R., LAROQUE G., Using privileged information to manipulate markets: Insiders, gurus and credibility, Quart. J. of Econ., 107, 1992, 921958.

[7] Bulow J., Klemperer P., Rational frenzies and crashes, J. of Pol. Econ., $102,1994,1-23$.

[8] CAPLIN A., LEHAY J., Business as usual, market crashes and wisdom after the fact, Amer. Econ. Rev., 84, 1994, 548-565.

[9] CENCI M., CERQUetTI A. M., Modelli evolutivi per un mercato azionario con valori fondamentali variabili nel tempo e asimmetria di informazioni, Atti XV Convegno Amases, Grado, 1991, 159-179.

[10] CoRnaglia A. et alii, Some models of imperfect financial markets, Quaderni dell'Istituto di Matematica Finanziaria dell'Università di Torino, serie III, n. 62, Torino, 1991.

[11] DaY R. H., HUANG W., Bulls, bears and market sheep, J. of Econ. Behav. and Org., 14, 1990, 299-329.

[12] De Long J. B., Shleifer A., Summers L. H., Waldmann R. J., Noise trader risk in financial markets, J. of Pol. Econ., 98, 1990, 703-738.

[13] De Long J. B., Shleifer A., Summers L. H., Waldmann R. J., Positive feedback investment strategies and destabilizing rational speculation, J. of Fin., 45, 1990a, 379-395.

[14] De Long J. B., Shleifer A., Summers L. H., Waldmann R. J., The survival of noise traders in financial markets, J. of Business, 54, 1991, $1-19$.

[15] GHEZZI L. L., Un modello non lineare sul funzionamento dei mercati azionari, Rivista di Matematica per le Scienze Economiche e Sociali, 15, 1992, 79-92.

[16] HARRISON J. M., KREPS D. M., Martingales and arbitrage in multiperiod securities markets., Journal of Economic Theory, 20, 1979, 381-408.

[17] Welch I., Sequential sales, learning, and cascades, J. of Fin., 47, 1992, 695-732.

\section{On the viability of a financial market}

\section{SUMMARY}

This work discusses a model of a financial market based on viability theory and differential inclusions. The model makes it explicit the existence of a relationship between the quantities of a security held by agents and its market price. Based on this, we show how to establish rigorously the existence of a time path for the price which in the literature is usually assumed without much justification. 